\title{
AN AUTOMATED GRAPHICAL USER INTERFACE BASED SYSTEM FOR THE EXTRACTION OF RETINAL BLOOD VESSELS USING KIRSCH'S TEMPLATE
}

\author{
Somnath Das ${ }^{1}$, Sandip Samaddar², Joshita Majumdar ${ }^{3}$ \\ ${ }^{1}$ Associate Professor, Department of Ophthalmology, Regional Institute of Ophthalmology Medical College, Kolkata. \\ ${ }^{2}$ Assistant Professor, Department of Ophthalmology, Regional Institute of Ophthalmology Medical College, Kolkata. \\ ${ }^{3}$ Department of Electronics \& Communication, IEM, WBUT, Kolkata.
}

\begin{abstract}
\section{BACKGROUND}

The assessment of blood vessel networks plays an important role in a variety of medical disorders. The diagnostic sign of Diabetic Retinopathy (DR) and other retinal vascular diseases including microaneurysms, haemorrhages, hard exudates and cotton wool spots is one such field. This study aims to develop an automated system for the extraction of blood vessels from retinal images by employing Kirsch's Templates in a MATLAB based Graphical User Interface (GUI).
\end{abstract}

\section{METHODS}

A RGB (Red, Green and Blue) or Grey image of the retina (Fundus Photography) is used to obtain the traces of blood vessels. We have incorporated a range of threshold values for the blood vessel extraction which would provide the user with greater flexibility and ease.

\section{RESULTS}

No expertise is required in this technique, even a technical or normal person can do this simply with a laptop or computer. Average diagnosis time is 5 to 10 seconds. The initial set up cost is 300 USD approximately and cost of procedure is practically nil.

\section{CONCLUSION}

This paper also deals with the more generalised implementation of various MATLAB (Matrix Laboratory) functions present in the image processing toolbox of MATLAB to create a basic image processing editor with different features like noise addition and removal, image cropping, resizing \& rotation, histogram adjust, separately viewing the red, green and blue components of a colour image along with brightness control, that are used in a basic image editor. We have combined both Kirsch's Template and various MATLAB Algorithms to obtain enhanced images which would allow the ophthalmologist to edit and intensify the images as per his/her requirement for diagnosis. The GUI contains simple and very commonly used English Language viz. Load, Colour Contrast Panel, Image Clarity, etc. It can incorporate maximum number of image processing techniques under one GUI to obtain higher performance. Also it would provide a cost effective solution towards obtaining high resolution images of retinal blood vessel in economically backward regions.

\section{KEYWORDS}

Blood Vessel Extraction; Retinal Images; Kirsch's Templates; Image Processing; Graphical User Interface.

HOW TO CITE THIS ARTICLE: Das S, Samaddar S, Majumdar J. An automated graphical user interface based system for the extraction of retinal blood vessels using Kirsch's template. J. Evolution Med. Dent. Sci. 2016;5(78):5828-5834, DOI: $10.14260 /$ jemds/2016/1315

\section{BACKGROUND}

The extraction of the human eye vasculature from retinal images involves the essential tool of edge detection. (1) According to Muthukrishnan \& Radha.[2], the classical methods of edge detection operates on convolving the image through an operator. Yin et al ${ }^{[3]}$ proposed a probabilistic tracking method to detect blood vessels in retinal images. They categorised the task of vessel extraction into two main groups: Pixel-based methods and tracking methods.

According to Zhang et al[4], matched filter is a simple yet effective method for vessel extraction. They also proposed an

Financial or Other, Competing Interest: None.

Submission 24-08-2016, Peer Review 17-09-2016,

Acceptance 23-09-2016, Published 29-09-2016.

Corresponding Author:

Dr. Sandip Samaddar

\# 25, Sarat Chandra Road,

East Barisha, Kolkata-700008.

E-mail: drsandipsamaddar@gmail.com

DOI: $10.14260 /$ jemds/2016/1315 extension to the matched filter approach to detect retinal blood vessels that significantly reduce the false detections produced by the original matched filter.

Esmaeili et al[5] presented an efficient algorithm for automatic extraction of blood vessels that comprises the following four steps: (i) Curvelet-based contrast enhancement, (ii) Matched filtering, (iii) Curvelet based edge-extraction and (iv) Length filtering. In their study, the enhanced image is first reconstructed from the modified curvelet co-efficient followed by match filtering to intensify the blood vessels along with the implementations of curvelet transform to segment vessels from its background. (6)

Finally, they have used length filtering to remove the misclassified pixels. Their experimental results have been evaluated on DRIVE database (Niemaiger \& van Ginneken. ${ }^{[7]}$ ). The images on which we worked on have been collected from a publicly available DRIVE database and from the Regional Institute of Ophthalmology, Medical College, Kolkata.

The next section describes the hardware \& software platforms of our system. Section III presents the methodology we proposed for blood vessel extraction. Sections IV \& V deal 
with the relevant discussions and Future Prospects respectively. The conclusion of the paper is presented in Section VI.

\section{HARDWARE AND SOFTWARE PLATFORMS}

Minimum Hardware Requirements

- Dual core processor.

- $\quad$ RAM: 4 GB.

- Windows XP/7/8.

- 256 MB Graphics card (to enhance the performance of the GUI).

\section{Our study has been conducted using}

- Laptop (SONY VAIO VPCEH16EN).

- Windows 7.

- $\quad$ Intel® Core $^{\mathrm{TM}} \mathrm{i} 3-2310 \mathrm{M}$ Processor $2.10 \mathrm{GHz}$.

- RAM : 4 GB.

- 256 MB NVIDIA GEFORCE ${ }^{\circledR}$ with CUDA

The software used to develop the GUI is MATLAB which is a multi-paradigm numerical computing environment and $4^{\text {th }}$ Gen. Programming language.

\section{METHODOLOGY}

The complete procedure of extracting the blood vessels from the coloured retinal image consists of three steps, beginning with fetching the input image from the system or the camera, then converting the RGB image into a gray scale image and finally using the Kirsch's templates to detect the edges of the blood vessels. (8) Fig. 1 illustrates the flow diagram of the proposed method for blood vessel extraction.

\section{Fetching the Image}

Two separate push buttons on the GUI facilitate the input of an image. One allows the user to browse an image from the system hard drive or any other external drives and the other allows the user to capture an image using any camera attached to the computer. The image that has been loaded is displayed on the first axis positioned on the top left corner of the GUI.

\section{Gray Conversion}

The panel 'Colour format' in the GUI contains a radio button 'GRAY Scale' that enables the user to convert the RGB retinal image to gray scale having pixel values ranging from 0 to 255 with just one mouse click.

\section{Extraction of Blood Vessels using Kirsch's Templates}

The Kirsch operator or Kirsch compass kernel is a non-linear edge detection that finds the maximum edge strength in a few predetermined directions. The 'VESSEL EXTRACTION' panel contains a popup menu with a number of threshold values (within a range of 4.6 to 9.0) for blood vessel extraction. The Kirsch operator can adjust the related threshold value automatically due to the image characteristics. The operator takes a single kernel mask and rotates it in $45^{\circ}$ increments through all eight compass directions: North, North-West, West, South-West, South, South-East, East and North-East as shown below:

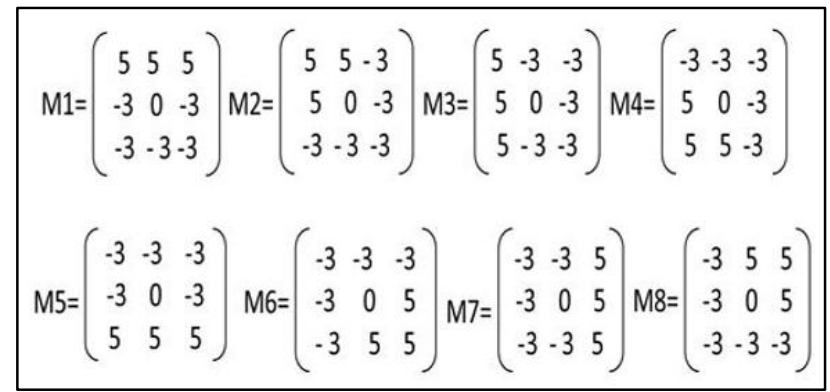

The edge magnitude of the Kirsch operator is calculated as the maximum magnitude across all directions. Except the outermost rows and columns, every pixel along with its eight neighbouring pixels in a given image is convolved with the eight aforementioned templates respectively.[9] providing eight outputs for each pixel, the maximum of which is defined as the edge magnitude (Gao et al[10] A pixel's Gray value with its eight neighbours is as shown).

\begin{tabular}{|lll|}
\hline $\mathrm{P}_{0}$ & $\mathrm{P}_{1}$ & $\mathrm{P}_{2}$ \\
$\mathrm{P}_{7}$ & $\mathrm{P}(\mathrm{i}, \mathrm{j})$ & $\mathrm{P}_{3}$ \\
$\mathrm{P}_{6}$ & $\mathrm{P}_{5}$ & $\mathrm{P}_{4}$ \\
\hline
\end{tabular}

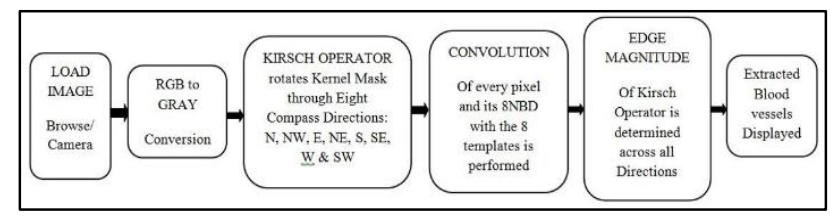

Fig. 1: Proposed Overall Method for Blood Vessel Extraction

The direction of edge is defined by the related mask that produces the maximum magnitude. The general output of edge detection through Kirsch's Templates is an image containing Gray level pixels of value 0 or 255 . The value 0 indicates a black pixel and the value 255 indicates a white pixel. The edge information of the target pixel is checked by determining the brightness levels of the neighbouring pixels.[11] In case no major difference in the brightness level is found, the possibility of the pixel being a part of an edge is ruled out.

Kirsch's Template can set and reset the threshold values to obtain most suitable edge of images. It works well for images having a clear distinction between the foreground and background.[12] Since the retinal blood vessels can be considered as the required foreground information from the background fundus images, Kirsch's algorithm is effectively applicable. Fig. 2 illustrates an original fundus image with the extracted blood vessels.

\section{Other Important Tools}

A fact worth mentioning is that the GUI designed in order to carry out this study on the extraction of blood vessels may also be used to edit and enhance the retinal images for further analysis using the following options.

- Image Addition.

- Image Adjust Panel.

- Pixel Processing, Image Rotate, Image Complement, Colour Swap. 
- Image Resize, Image Crop.

- Contrast Slider, R G B Sliders.

- CMY Panel.

- $\quad$ Colour Panel - Gray, B \& W, Pseudo.

- $\quad$ Noise Addition, Filter.

- Edge Detection.

- $\quad$ B \& W Morphological Operations.

- Colour Plane Models.

- Hue.

- Saturation.

- Value.

- HSV.
- Luma.

- Image Save, etc.

\section{SOME OF THESE FEATURES HAVE BEEN DISCUSSED BELOW}

1. Image Addition

Image is a simple matrix. Since addition can be performed on matrix, that can also be applied on images. To do this, a function available in MATLAB is used which performs addition of pixel values of first image to the second image. Addition of the image by itself gives a better clarity of the original image. Fig. 3 demonstrates the image addition operation.

\section{Image Clarity}

Generally, for certain images, the pixel distribution is not equally spaced or rather clotted to a particular intensity level, hence, making the image too dull or too bright.[13] For this reason, the following histogram adjustment techniques are being used: Image Adjust, Histogram equalisation, Adaptive Histogram equalisation.
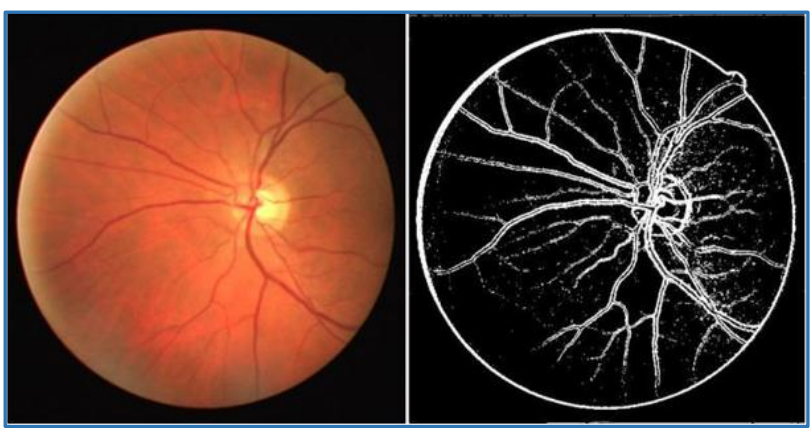

Fig. 2: Extracted Blood Vessels

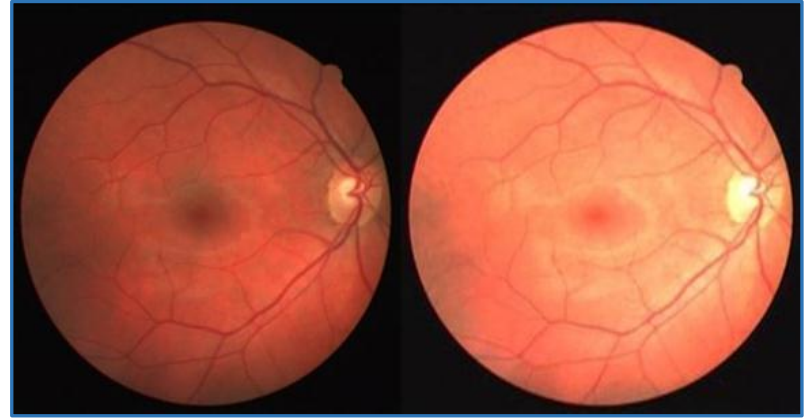

Fig. 3: Demonstration of Addition Operation on the Image

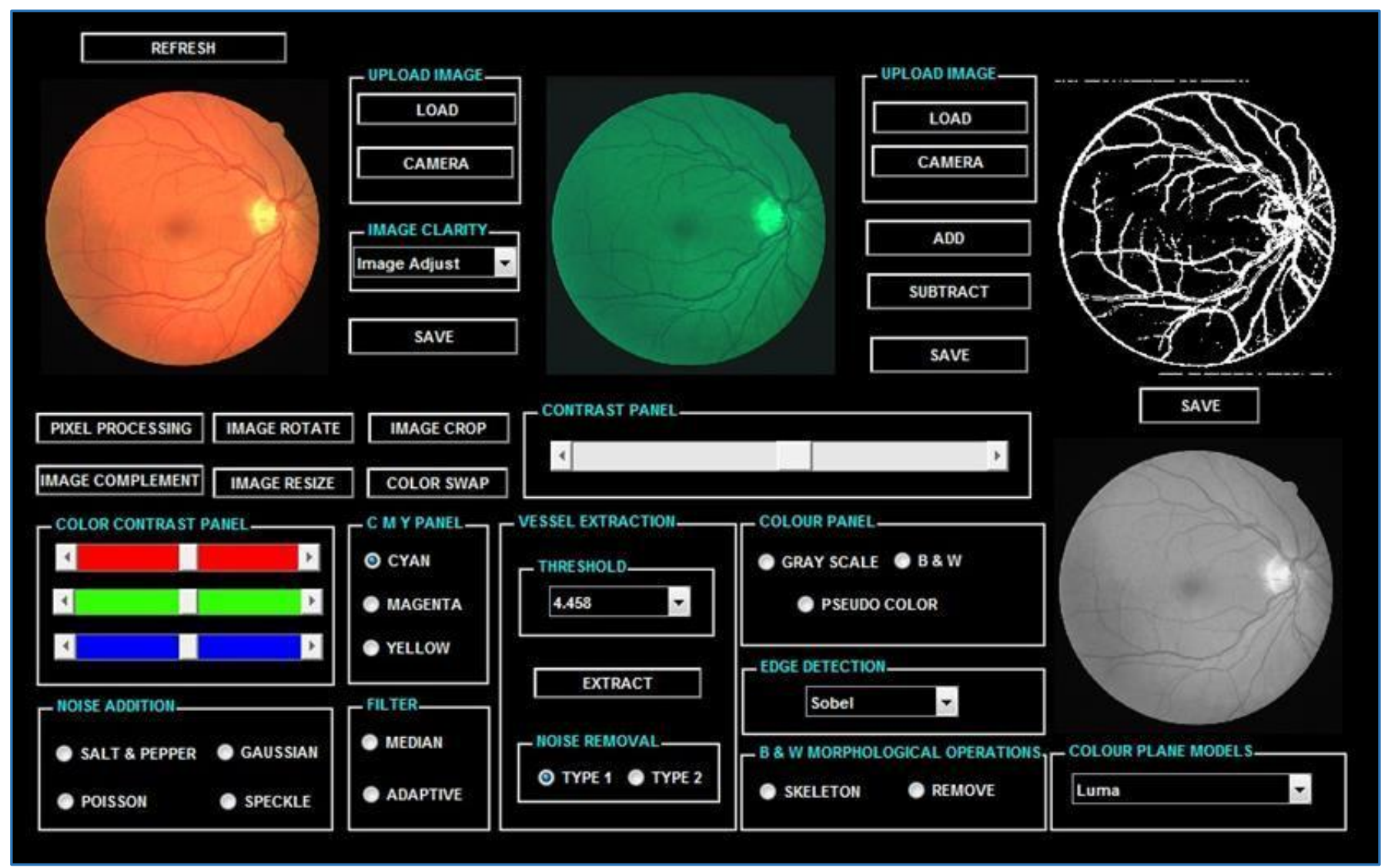

Fig. 4: Complete MATLAB based GUI demonstrating the Extraction of Retinal Blood Vessels Using Kirsch's Templates 


\section{Image Rotate}

Image rotation is used to rotate the image to a specified degree and get a different perspective of view.

Image Complement: Image complement simply complements the image. Here, the RGB image of the fundus is complemented where each pixel value is subtracted from the maximum pixel value supported by the class and the difference is used as the pixel value in the output image.

Image Resize: It is being used to resize the actual image to certain multiples.

Image crop: It is used to select any particular portion of the whole image.

Contrast Panel: This panel is used to apply brightness or contrast to the image. It is varied over a range of 0 to 255 .

Colour Contrast Panel: Slider for Red, Green and Blue are added to view the single coloured image in varied brightness (dark and light). It has been mentioned that a colour or RGB image is an overlap of three two dimensional matrices.

CMY Panel: This panel is used to view the Cyan, Magenta and Yellow components of the image separately.

Colour Panel: The most important feature of this panel is the 'GRAY' radio button that converts an RGB image into a Gray scale image.

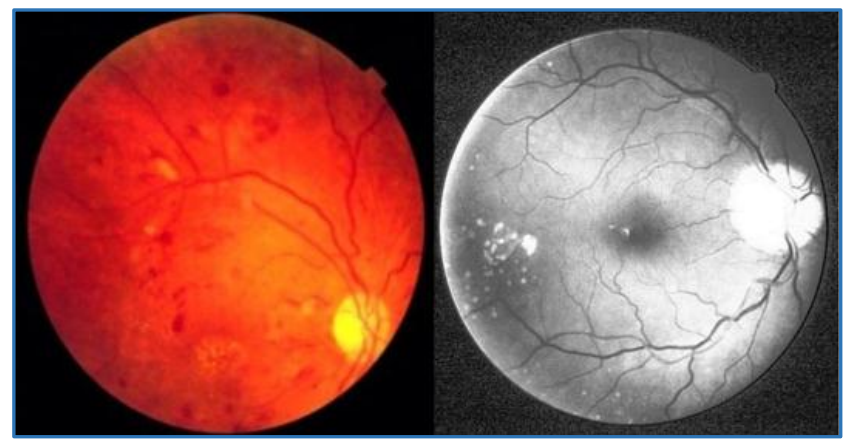

Fig. 5: Image Adjust and Histogram Equalisation

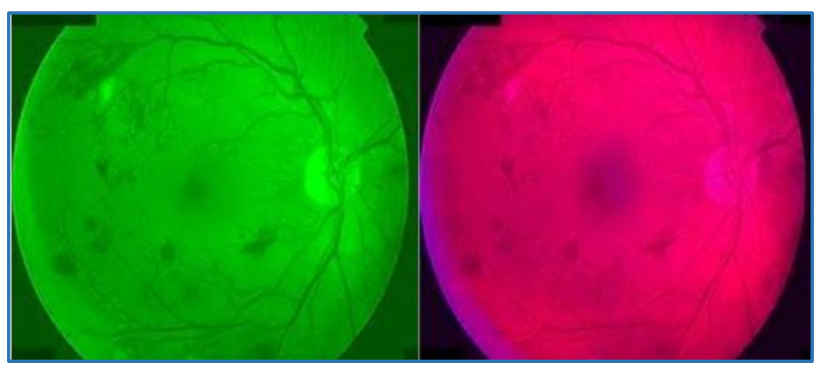

Fig. 6: Green \& Magenta Images in addition with Contrast

\section{Noise addition and Removal}

Various types of noises get added to an image when a snapshot is taken. In order to get rid of these noises various types of filters are used. To illustrate this, we have added a noise to an image externally and then applied various filters to get rid of it and evaluated the results. The types of noises available in this GUI are Poisson, Salt \& Pepper, Gaussian and Speckle. Noise removal maybe performed using either a Median filter or an Adaptive filter. Fig. 9. Shows the addition and removal of 'salt and pepper noise'.

\section{Edge Detection}

The various types of edge detection techniques are: Sobel, Prewitt, Roberts, LoG, Canny and Zero cross. These options are available for the user to compare the results with those of the Kirsch's Algorithm.

\section{Colour Plane Models}

In this panel, the ophthalmologist would get the opportunity to select any of the following image type which would enable a different cylindrical-coordinate representation of the RGB Colour image:

a) Hue image: Using this option might enable the ophthalmologist to detect further abnormalities of the fundus $\left[{ }^{[14]}\right.$ that are not very evident from the Retinal Blood Vessel Extraction.

b) Saturation image: This option would let the ophthalmologist view the picture of the fundus in a different colour tone as shown in Fig. 8.

c) Hue Saturation Value (HSV) Image: The combination of Hue Saturation and Value gives a better outlook of the fundus for detection of abnormalities.

d) Luminosity (luma) Image: By this option the ophthalmologist gets the view of the weighted sum of the gamma corrected image of the main RGB image of the fundus

Save: The Save option is provided against the three working axes present in the middle, top right corner and bottom right corner of the GUI for the purpose of saving the edited or worked on images for future reference.

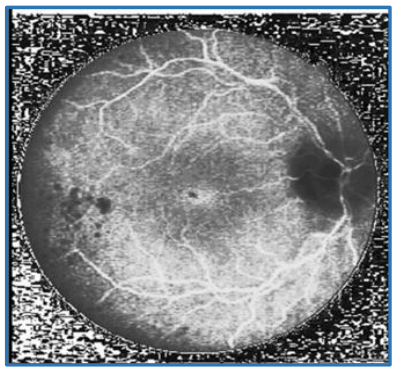

Fig. 7: Hue Image
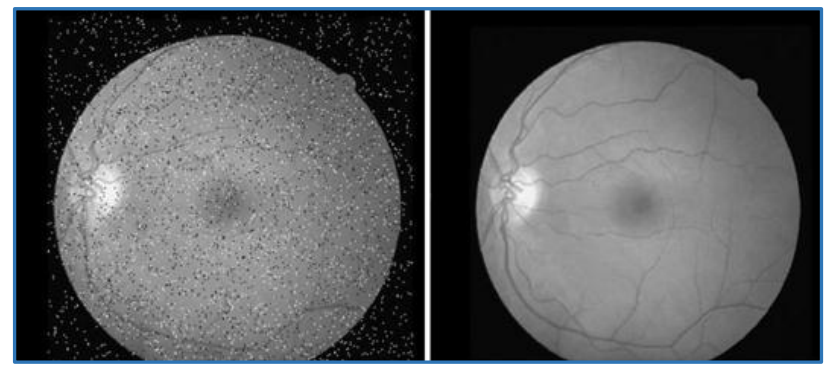

Fig. 9: Demonstration of Addition and Removal of 'Salt \& Pepper' noise using a Median Filter

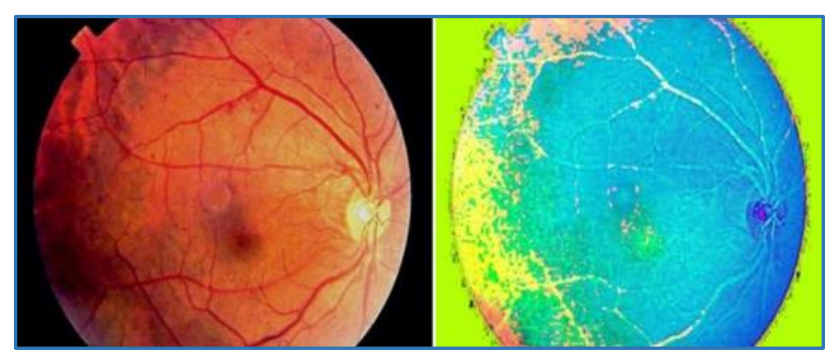

Fig. 10: Demonstration of HSV Image in Comparison to the original Funduscopic Image 


\section{DISCUSSIONS}

Diabetic Retinopathy and other retinal vascular diseases are important cause of blindness(5) due to damage caused to the retinal blood vessels. We have presented in this paper the retinal blood vessel extraction with the help of Kirsch's Templates and a combination of various MATLAB image processing techniques. The combination of these two techniques provided an optimum result. Table 1 depicts the comparison between our Results and other Techniques in the extraction of Blood Vessels.
Our main aim is to provide a cost effective solution to detect Retinal anomalies and provide a better platform. In some cases, minute examination of vascular Retinopathy is carried on and analysed by Fundus Photography and for detailed insight, Optical Coherence Tomography is used.[16] Table 2 represents a comparative study between our Graphical User Interface and the present clinical technique for the detection of Retinal diseases.

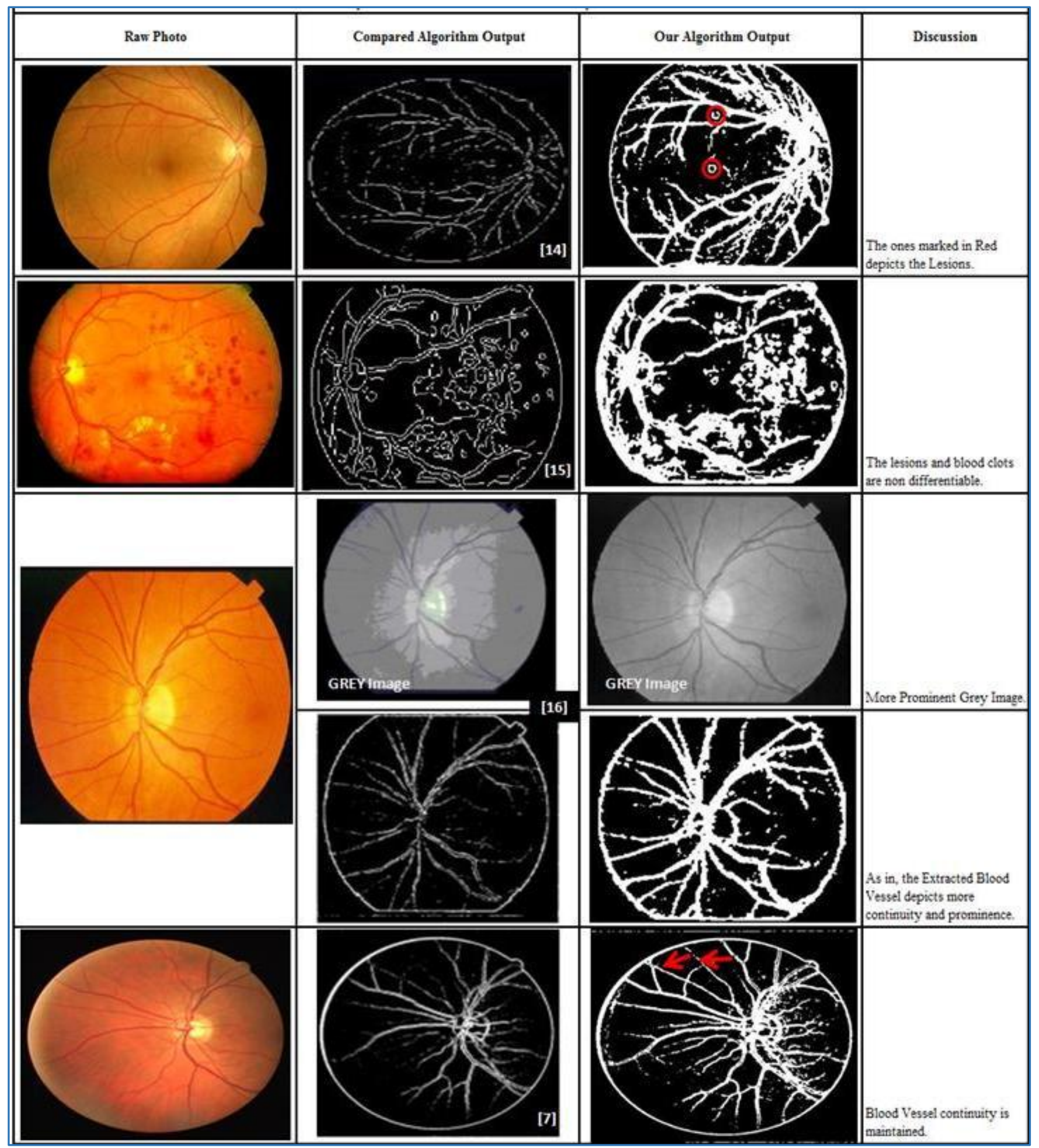

Table 1: Comparison of our Results with other Techniques for Blood Vessel Extraction 


\begin{tabular}{|c|c|c|}
\hline $\begin{array}{c}\text { Fundus Photography + Our Graphical User } \\
\text { Interface }\end{array}$ & \multicolumn{2}{|c|}{ Fundus Photography + Optical Coherence } \\
\cline { 2 - 3 } Target Audience & $\begin{array}{c}\text { Anyone (A super specialised Doctor } \\
\text { or even a Normal Person ) }\end{array}$ & Doctor or Experienced Technician \\
\hline Ease of Use & No Expertise required & Expertise required \\
\hline Diagnosis Time & $5-10$ seconds & Few minutes \\
\hline Initial Setup & A Computer/Laptop & The OCT machine \\
\hline Initial Setup Cost & 300 USD (approx.) & Diagnosis Charge \\
\hline Cost & NIL & (approx.) \\
\hline \multicolumn{2}{|c|}{} \\
\hline \multicolumn{2}{|c|}{ Table 2: Comparison of our Graphical user Interface with present Methodology for Diagnosis of Retinal Abnormalities } \\
\hline
\end{tabular}

This automated system for the extraction of Retinal blood vessels can give us important clue regarding alteration in morphological pattern and pathological changes in and around the retinal blood vessels, status of laminar blood flow within the blood vessels and nature of extravasations of plasma, blood cells and lipids in surrounding retinal tissue on the basis of which we can diagnose and plan for the management of a large group of ocular diseases. Not only that, this system will also be a good prognostic indicator for a particular disease".

Availability of a Computer/Laptop will enable the user to use the Graphical User Interface after a quick installation of MATLAB.

One of the limitations of this Graphical User Interface is that it requires a high processor speed for smoothness and trouble-free output. Other than that, slight lag time can be experienced during the execution of the program.

\section{Future Prospects}

As an Initial stage of our work, we have chosen Diabetic Retinopathy as our base to validate the Graphical User Interface performance. We can also utilise our Graphical User Interface to understand pathogenesis and ongoing changes in the retinal blood vessels in diseases like Diabetic Retinopathy, Central Retinal Vein Occlusion(CRVO), Branch Retinal Vein Occlusion (BRVO), Central Retinal Artery Obstruction (CRAO), Hypertensive Retinopathy by which we can make staging of the disease can assess the severity or actual burden of the disease and can make a suitable management protocol to treat it (LASER photocoagulation, intra-vitreal injection of antivascular endothelial growth factor, etc.). We can also detect and evaluate diseases like acquired immunodeficiency syndrome related Retinopathy, Arteriovenous malfunctions, Optic Atrophy, Ocular diseases like Ocular Ischaemic syndrome, Arterio-venous malformation in carotid-cavernous fistula and haemangioma, TORCH infections having an appearance of CMV (Cytomegalovirus) Retinitis, Salt \& Pepper Retinopathy, Rubella, etc. with the help of our Graphical User Interface. Knowledge about vascular stasis and detection of abnormal optic nerve head perfusion pressure can be extremely helpful as far as the diagnosis of Open angle Glaucoma, Normal tension glaucoma and retinal vasculitis are concerned. Comparison of structural pattern of retinal vascular tree in Eales' disease, Panuveitis, Posterior Uveitis and Takayasu's disease involving retina can be done with this method.

Furthermore, we can incorporate an algorithm to differentiate between blood clots and lesions and provide a comparative study. This would help in distinguishing various retinal abnormalities. (17) But for all this, we require the fundus photography which is obtained by a fundus camera which costs almost 3600 USD. To cut down the cost at that level, the next step could be utilising the Smart Phone's Camera and combining it with an extra lens to capture Fundus images more easily and in a very low cost. This whole setup can then be incorporated in Smart phones as an Application which will be easily accessible and utilised by all. Further classification of special features and parameters detected from the extracted blood vessels along with the use of a trained Probabilistic Neural Network to recognise and report any of the abovementioned abnormalities can be carried out automatically. [1]

\section{CONCLUSIONS}

Retinal images are being used by ophthalmologists to aid in diagnosis, to make measurements, and to look for changes in lesions or severity of diseases. The appearance of the retinal vasculature particularly acts as an indicator for diagnosis of Diabetic Retinopathy (both Proliferative and NonProliferative) and Glaucoma. Therefore, extraction of these features is the key challenge for proper analysis, visualisation and quantitative comparison. The present study focuses mainly on this challenge of blood vessel extraction from colour retinal images obtained from funduscopy. The proposed algorithm proves to be successful and robust in accurately extracting the retinal blood vessels. In this respect, the dataset of 40 test images from the DRIVE database has been used to evaluate this method. Few real time images obtained from the Regional Institute of Ophthalmology (RIO), Medical College, Kolkata are also used for the evaluation.

It included funduscopy of various patients having NPDR (Non-proliferative Diabetic Retinopathy), PDR (Proliferative Diabetic Retinopathy) and post PRP (Panretinal Photocoagulation). An accurate extraction of blood vessels provides the basis for diagnosis, treatment, evaluation and clinical study.[18] More particularly in rural areas of underdeveloped and developing countries, the proposed Graphical User Interface can be utilised to overcome the hardships arising due to the shortage of professional observers by this completely automated computer assisted monitoring and diagnostic system.

\section{REFERENCES}

1 Muthukrishnan R, Radha M. Edge detection techniques for image segmentation. International Journal of Computer Science \& Information Technology (IJCSIT) 2011;3(6):259-67.

2 Yin Y, Adel M, Bourennane S. Retinal vessel segmentation using a probabilistic tracking method. Pattern Recognition 2012;45(4):1235-44.

3 Zhang B, Zhang L, Zhang L, et al. Retinal vessel extraction by matched filter with first-order derivative of Gaussian. Computers in Biology and Medicine 2010;40(4):438-45.

4 Esmaeili M, Rabbani H, Mehri A, et al. Extraction of retinal blood vessels by curvelet transform. 16th IEEE International Conference on Image Processing (ICIP). 
2009:3353-56.

5 Niemeijgr M, Staal J, van Ginneken B, et al. Comparative study of retinal vessel segmentation methods on a new publicly available database. SPIE Medical Imaging 2004;5370:648-56.

6 Karasulu B. Automatic extraction of retinal blood vessels: a software implementation. European Scientific Journal 2012;8(30):47-57.

7 Gao P, Sun X, Wang W. Moving object detection based on kirsch operator combined with optical flow. International Conference on Image Analysis and Signal Processing (IASP) 2010;9(11):620-4.

8 Vijayakumari V, Suriyanarayanan N. Survey on the detection methods of blood vessels in retinal images. European Journal of Scientific Research 2012:68(1). ISSN 1450-216X.

9 Kirbas C, Quek F. A review of vessel extraction techniques and algorithms. ACM Computing Surveys 2004;36(2):81121.

10 Buksh R, Routh S, Mitra P, et al. MATLAB based image editing and color detection. International Journal of Scientific and Research Publications 2014;4(1):1-6.

11 Hayashi J, Kunieda T, Cole J, et al. A development of computer-aided diagnosis system using fundus images. In: proceeding of the seventh international conference on Virtual Systems and Multi Media (VSMM) 2001:429-38.

12 Global data on visual impairments 2010. Geneva, World Health Organization, 2012.
13 Kavitha M, Palani S. A new fast curvelet transform with morphological operations based method for extraction of retinal blood vessels using graphical user interface. International Journal of Scientific \& Engineering Research 2012;3(6):1-6.

14 Sharma A, Hempriya. Detection of blood vessels and diseases in human retinal images. International Journal of Computer Science and Communication Engineering. IJCSCE Special issue on Emerging Trends in Engineering \& Management ICETE 2013:9-11. ISSN 2319-7080.

15 Bhadauria HS, Bisht SS, Singh A. Vessels extraction from retinal images. IOSR Journal of Electronics and Communication Engineering (IOSR-JECE) 2013;6(3): 79-82.

16 Browning DJ, McOwen MD, Bowen RM, et al. Comparison of the clinical diagnosis of diabetic macular edema with diagnosis by optical coherence tomography. Presented at: American academy of ophthalmology annual meeting, November, 2003, Anaheim, California. Ophthalmology 2004;111(4):712-5.

17 Bhagapathi B, Das C. Edge detection of digital images using fuzzy rule based technique. International Journal of Advanced Research in Computer Science and Software Engineering 2012;2(6):259-62.

18 Osareh A, Shadgar B. Automatic blood vessel segmentation in color images of retina. Iranian Journal of Science \& Technology, Transaction B, Engineering 2009;33(B2):191-206. 